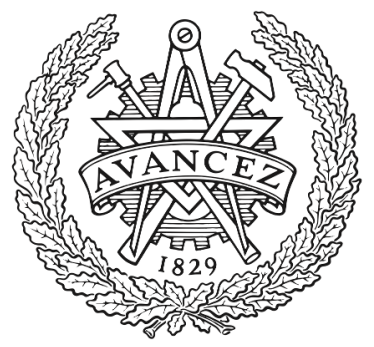

CHALMERS

UNIVERSITY OF TECHNOLOGY

\title{
An impairment-aware resource allocation scheme for dynamic elastic optical networks
}

Downloaded from: https://research.chalmers.se, 2023-04-26 14:30 UTC

Citation for the original published paper (version of record):

Dharmaweera, M., Yan, L., Karlsson, M. et al (2017). An impairment-aware resource allocation scheme for dynamic elastic optical networks. Optical Fiber Communication Conference (OFC) Proceedings, Part F40. http://dx.doi.org/10.1364/OFC.2017.Th2A.19

N.B. When citing this work, cite the original published paper. 


\title{
An impairment-aware resource allocation scheme for dynamic elastic optical networks
}

\author{
Nishan Dharmaweera ${ }^{1}$, Li Yan ${ }^{2}$, Magnus Karlsson ${ }^{1}$, and Erik Agrell ${ }^{2}$ \\ ${ }^{1}$ Photonics Laboratory, Department of Microtechnology and Nanoscience, ${ }^{2}$ Department of Signals and Systems, \\ Chalmers University of Technology, SE-412 96 Gothenburg, Sweden \\ nishan@chalmers.se
}

Abstract: By using impairment-driven variable guardbands, our proposed dynamic resource allocation scheme accommodates 50\% more traffic in comparison to existing fixed transmission-reach- and guardband-based algorithms.

OCIS codes: (060.4510) Optical communications, (060.4256) Networks, network optimization

\section{Introduction}

Existing studies show that physical-layer impairments (PLI) have a significant effect on the performance of elastic optical networks (EONs) [1,2]. In most routing, modulation, and spectrum assignment (RMSA) schemes designed for EONs with dynamic traffic, impairments are handled by setting predefined transmission-reach (TR) limits [3,4] for different modulation schemes and by placing a fixed-size guardband (GB) between connections [5-7]. However, as highlighted in [2], predefined TRs and a fixed-size GB do not consider the actual network state, and, thus, may over- or underestimate nonlinear impairments (NLI). Consequently, these TR-and GB-based RMSA schemes may not provide the best performance. In our previous work $[2,8]$, we showed that by accurately accounting for impairments using the Gaussian Noise (GN) model in EONs with static traffic, spectral efficiency can be significantly improved over existing TR- and GB-based RMSA schemes. Extending the work in [2] and [9], in the current study, we analyze the performance improvements that can be achieved in EONs serving dynamic traffic.

\section{Impairment model and problem statement}

The PLI model and values of the relevant parameters are adopted from [8]. The quality of a transmitted connection degrades due to the accumulation of amplified spontaneous emission (ASE) noise and NLI caused by self-channel interference (SCI) and cross-channel interference (XCI). To ensure the quality of transmission (QoT) of any connection $i$, the signal-to-noise ratio of $i\left(\mathrm{SNR}_{i}\right)$ must be larger than or equal to the SNR threshold of the assigned modulation format $c\left(\mathrm{SNR}^{c}\right)$. The set of available modulation formats $M=$ [PM-QPSK, PM-8QAM, PM-16QAM], their respective spectral efficiency $c$, and the SNR thresholds $\mathrm{SNR}^{c}$ were adopted from our previous work.

Given a network with a set of nodes $V$, set of links $E$, and a dynamic traffic matrix, the goal of our proposed RMSA scheme is to accommodate a maximum number of connections while satisfying the spectrum continuity, contiguity, and QoT constraints.

\section{Proposed heuristic}

Our proposed scheme serves every connection $i$ by sequentially running through two stages. The bit rate, holding time, $\mathrm{K}$-shortest routing paths, bandwidth at modulation format $m$, number of used spectrum slots, and size of the assigned GB of connection $i$ are represented as $b_{i}, t_{i}, P_{i}, \Delta f_{i m}, Q_{i}$, and $\mathrm{GB}_{i}$, respectively. While $H$ represents the available GB sizes $(H=[1, \ldots, n]), Z_{r}$ denotes the unoccupied common spectrum slots in fibers of route $r$.

In stage 1, which is summarized in Algorithm 1, our scheme identifies different combinations of routing paths, modulation formats, GB sizes, and respective spectrum segments that could be used to accommodate connection $i$, which are stored in the list $L_{i}$. Algorithm 1 begins its search by sequentially running through the candidate routing paths and modulation formats.

For every route $r$ and modulation format $m$, the bandwidth $\Delta f_{i m}$ and the number of slots $Q_{i}$ are first calculated. Then, the PLI due to ASE and SCI are calculated. Connection $i$ incurs XCI from both existing ( $\mathrm{X}_{\text {Existing }}$ ) and future connections. However, as the controller is only aware of the current network status, the scheme approximates the $\mathrm{XCI}$ incurred by future connections (FuCons) using the following assumptions: the number of FuCons $N$ is found by multiplying the average traffic arrival rate $R$ and the connection holding time $t_{i}$, the bit rate of a FuCon $U$ is identical to the average connection bit rate of the network, the number of slots used by a FuCon $O$ is found by modulating $U$ with the lowest order modulation format, and the $N$ FuCons are placed as a contiguous set next to the highest frequency slot of connection $i$ in the fibers along route $r$. The spacing between connection $i$ and the set of FuCons are determined by the size $g$ of the GB. The XCI for different-sized GBs $X_{\text {Approx }}^{g}$ are calculated accordingly. Using first-fit assignment, the scheme then identifies an unoccupied spectrum segment (with indices $\left[s, \ldots,\left(s+Q_{i}+g-1\right)\right]$ ) to accommodate 
connection $i$ on $r$, using $m$, and GB size $g$, which satisfy the constraints mentioned previously. The function CheckPLI returns 1 if all established connections satisfy QoT constraints when connection $i$ is accommodated on $r, m, g$, and spectrum segment $\left[s, \ldots,\left(s+Q_{i}+g-1\right)\right]$, else it returns 0 .

Finally, the length of the chosen route $r$, spectral efficiency, and the SNR threshold of the modulation format $m$, the GB size $g$, the last slot index of the assigned spectrum $s+Q_{i}+g-1$, and the value of $\mathrm{SNR}_{i}$ are expressed as $\mathrm{LEN}_{e^{j}}$, $\mathrm{MOD}_{e^{j}}, \mathrm{TH}_{e^{j}}, \mathrm{GBS}_{e^{j}}, \mathrm{IND}_{e^{j}}$, and $\mathrm{QOT}_{e^{j}}$, respectively, and are stored in an entity $e^{j}$. By repeating this process for $\forall r \in P_{i}, \forall m \in M$, and $\forall g \in H$, the scheme produces the populated list $L_{i}=\left[e^{1}, e^{2}, . . e^{n}\right]$.

Stage 2 is described in Algorithm 2. Here, the best entity in list $L_{i}$ - denoted as $D_{i}$ - that offers superior QoT performance and spectral efficiency is selected and connection $i$ is accommodated accordingly. Thus, an entity that is most tolerant to impairments is selected. If two or more entities are equally tolerant, then a higher modulation format, shorter transmission distance, or smaller subcarrier index are used sequentially as tiebreakers. If the list is empty, then the connection is blocked. The tolerance to impairments of an entity $e^{j}, \mathrm{TOL}_{e^{j}}$, is estimated to be $\mathrm{QOT}_{e^{j}} / \mathrm{TH}_{e^{j}}$.

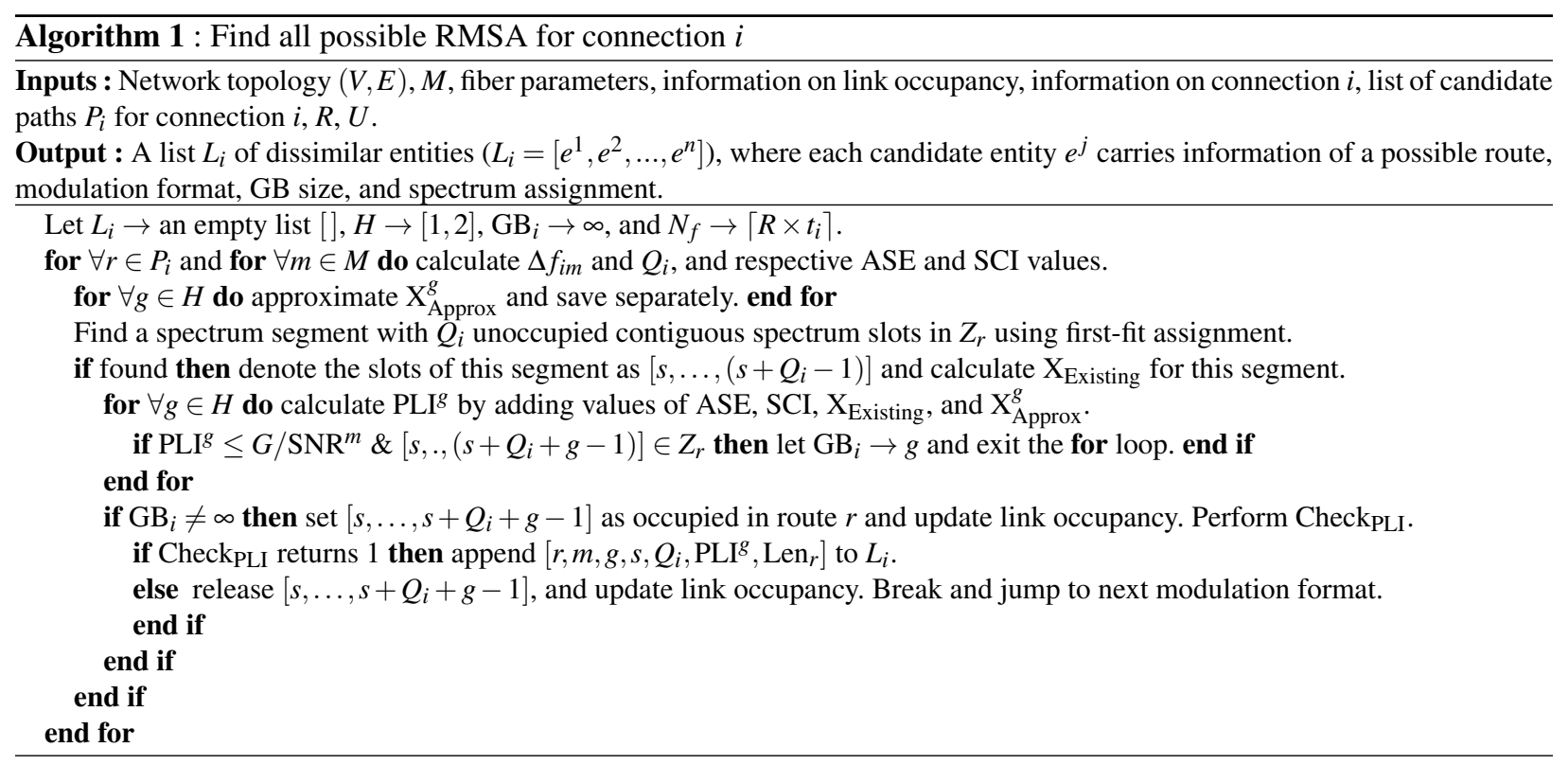

\section{Performance evaluation}

The results of the proposed RMSA scheme were compared against the benchmark TR- and GB-based QoT-aware algorithm in [7]. Specifically, we consider the KSPC-MDPS-FFSA (K-shortest-path computation with most dominant path selection and first-fit spectrum assignment) presented in [7] as the benchmark for our work. In our opinion, this algorithm [7] is more accurate than other RMSA schemes as it accounts for impairments using a more accurate analytical model [10]. Although slightly better results were obtained in [7] by using the combination MD-PC (modified path computation) and TB-SA (traffic balancing-spectrum assignment), it has a higher computational complexity than the KSPC-MDPS-FFSA alone.

The simulations were performed on the Deutsche Telekom (DT) and the National Science Foundation (NSF) networks [11]. The bandwidth of every fiber is divided into 320 spectrum slots of $12.5 \mathrm{GHz}$ each. In the dynamic traffic model, connections between random node-pairs arrive following a Poisson process and, thus, traffic load is quantified in Erlangs. The bit rate of every connection is randomly picked from a uniformly distributed range of 150-500 Gbps. To guarantee that the obtained results are tolerant to traffic variations, for each Erlang value, average results are reported after repeating the simulation with 40 different traffic distributions. A uniform PSD $G=20 \mathrm{~mW} / \mathrm{THz}$ was assumed for all connections. The benchmark algorithm was tested separately for a fixed-size GB of 0 slot (B0), 1 slot (B1), and 2 slots (B2). The performance of the proposed RMSA scheme (denoted as PRMSA) and the benchmark algorithm are measured in terms of connection blocking ratio (CBR) and throughput in Figs. 1a and 1b, respectively.

The results in Fig. 1a clearly indicate that PRMSA blocks fewer connections in both the DT and NSF networks and, on average, accommodates up to 50\% more connections in the larger NSF network. However, in the DT network, the PRMSA and B0 offer similar performance at low traffic loads, the reason being that since the PLI is directly related to transmission distance, which is much shorter in the DT network, a GB size of 0 slots is adequate to satisfy the QoT of most connections at low traffic loads. For the large NSF network, PRMSA offers better CBR performance at 


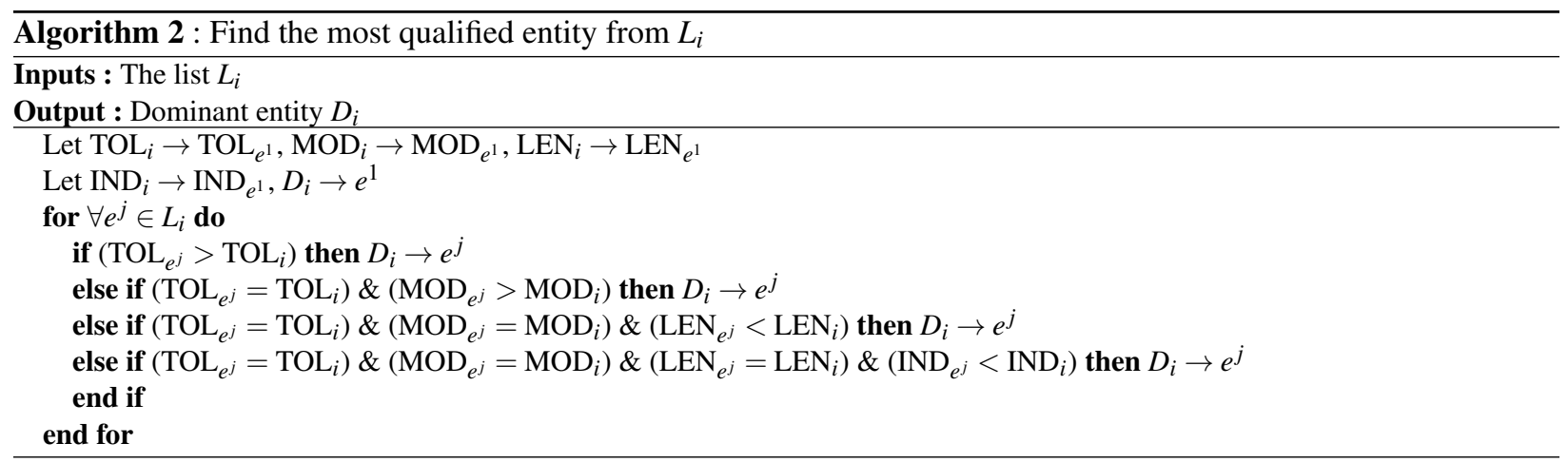

higher traffic loads, but drops more connections when traffic is significantly small $(R \approx 0)$ as it does not accurately estimate the number of future connections. The benchmark algorithm is oblivious to future connections and, therefore, in some instances it could accommodate the "killer connection" that makes fibers along its route unusable for future connections. These results show that there is no one-size-fits-all solution to the GB problem for different networks. The results in Fig. 1b show that while the difference in throughput is minimal for the DT network, the PRMSA offers over $100 \%$ improvement in the larger NSF network.
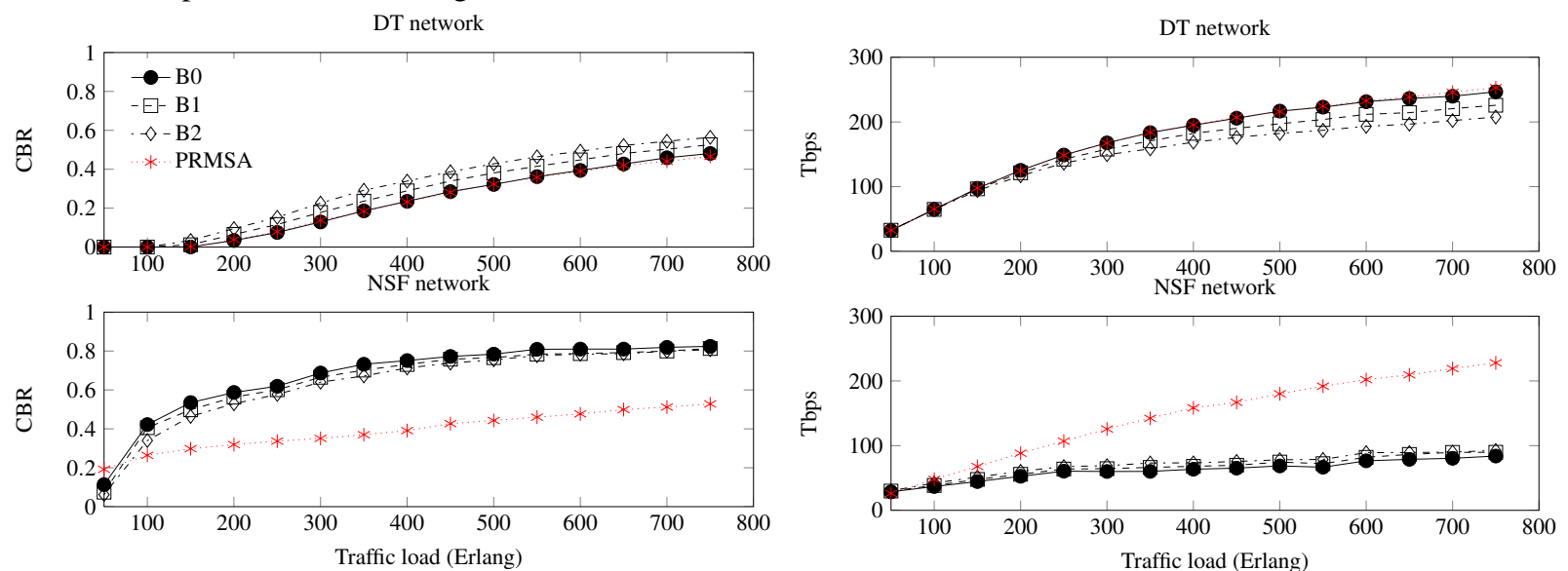

(a)

(b)

Fig. 1: The connection blocking ratio (CBR) (a) and achieved throughput (b) vs. increasing traffic load for the DT and NSF networks by the benchmark algorithm with respective GB sizes (B0, B1, and B2) and the proposed algorithm (PRMSA).

\section{Conclusion}

By appropriately selecting the size of the guardband assigned to each connection based on accurate impairment estimations, our proposed scheme reduces the number of dropped connections by $50 \%$ and achieves over a $100 \%$ increase in throughput over fixed TR- and GB-based RMSA schemes for large dynamic EON networks.

\section{References}

1. K. Christodoulopoulos et al., "Elastic bandwidth allocation in flexible OFDM-based optical networks," JLT, vol. 29, pp. 1354-1366, 2011.

2. J. Zhao et al., "Nonlinear impairment-aware static resource allocation in elastic optical networks," JLT, vol. 33, pp. 4554-4564, 2015.

3. T. Takagi et al., "Dynamic routing and frequency slot assignment for elastic optical path networks that adopt distance adaptive modulation," in $O F C, 2011$, pp. OTuI7.

4. X. Wang et al., "Dynamic routing and spectrum allocation in elastic optical networks with minimized line rates," JOCN, vol. 6, pp. 1115$1126,2014$.

5. J. Zhang et al., "Dynamic traffic grooming in sliceable bandwidth-variable transponder-enabled elastic optical networks," JLT, vol. 33, no. 1, pp. 183-191, 2015.

6. L. Velasco et al., "Solving routing and spectrum allocation related optimization problems: from off-line to in-operation flexgrid network planning," JLT, vol. 32, pp. 2780-2795, 2014.

7. H. Beyranvand and J.A. Salehi, "A Quality-of-transmission aware dynamic routing and spectrum assignment scheme for future elastic optical networks," JLT, vol. 31, pp.3043-3054, 2013.

8. L. Yan et al., "Resource allocation for flexible-grid optical network with nonlinear channel model," JOCN, vol. 7, pp. B101-B108, 2015.

9. J. Zhao et al., "Nonlinear impairment aware resource allocation in elastic optical networks," in $O F C$, 2015, pp. M2I.1.

10. G. Gao et al., "Analytical expressions for nonlinear transmission performance of coherent optical OFDM systems with frequency guard band," JLT, vol. 30, pp. 2447-2454, 2012

11. R. Hulsermann et al., "A set of typical transport network scenarios for network modelling," in 5th ITG Workshop on Photonic Networks, pp. 65-71 (2004). 